\title{
Evaluation of pre-hospital administration of adrenaline (epinephrine) by emergency medical services for patients with out of hospital cardiac arrest in Japan: controlled propensity matched retrospective cohort study
}

\author{
(c) (1) (8) OPEN ACCESS
}

Shinji Nakahara assistant professor ${ }^{1}$, Jun Tomio assistant professor ${ }^{23}$, Hideto Takahashi associate professor $^{4}$, Masao Ichikawa professor ${ }^{4}$, Masamichi Nishida director ${ }^{5}$, Naoto Morimura professor ${ }^{6}$, Tetsuya Sakamoto professor ${ }^{7}$

${ }^{1}$ Department of Epidemiology and Health Promotion, Saint Marianna University School of Medicine, Kawasaki, Japan; ${ }^{2}$ Department of Public Health, Graduate School of Medicine, University of Tokyo, Tokyo, Japan; ${ }^{3}$ Department of Disaster Medical Management, University of Tokyo Hospital, Tokyo; ${ }^{4}$ Faculty of Medicine, University of Tsukuba, Tsukuba, Japan; ${ }^{5}$ Department of Emergency Medicine, Toranomon Hospital, Tokyo, Japan; ${ }^{6}$ Department of Emergency Medicine, Yokohama City University Graduate School of Medicine, Yokohama, Japan; ${ }^{7}$ Department of Emergency Medicine, Teikyo University School of Medicine, Tokyo, Japan

\begin{abstract}
Objectives To evaluate the effectiveness of pre-hospital adrenaline (epinephrine) administered by emergency medical services to patients with out of hospital cardiac arrest.

Design Controlled propensity matched retrospective cohort study, in which pairs of patients with or without (control) adrenaline were created with a sequential risk set matching based on time dependent propensity score.

Setting Japan's nationwide registry database of patients with out of hospital cardiac arrest registered between January 2007 and December 2010.

Participants Among patients aged 15-94 with out of hospital cardiac arrest witnessed by a bystander, we created 1990 pairs of patients with and without adrenaline with an initial rhythm of ventricular fibrillation or pulseless ventricular tachycardia (VF/VT) and 9058 pairs among those with non-VF/VT.
\end{abstract}

Main outcome measures Overall and neurologically intact survival at one month or at discharge, whichever was earlier.

Results After propensity matching, pre-hospital administration of adrenaline by emergency medical services was associated with a higher proportion of overall survival (17.0\% v 13.4\%; unadjusted odds ratio $1.34,95 \%$ confidence interval 1.12 to 1.60 ) but not with neurologically intact survival (6.6\% v6.6\%; $1.01,0.78$ to 1.30$)$ among those with VF/VT; and higher proportions of overall survival (4.0\% v2.4\%; odds ratio 1.72 , 1.45 to 2.04$)$ and neurologically intact survival $(0.7 \% \vee 0.4 \% ; 1.57,1.04$ to 2.37 ) among those with non-VF/VT.

Conclusions Pre-hospital administration of adrenaline by emergency medical services improves the long term outcome in patients with out of hospital cardiac arrest, although the absolute increase of neurologically intact survival was minimal.

\section{Introduction}

Pre-hospital cardiopulmonary resuscitation for patients with out of hospital cardiac arrest commonly includes administration of adrenaline (epinephrine) by emergency medical services. Despite extensive research on its effectiveness, there is no definite evidence to support its routine use. Whether pre-hospital use of adrenaline improves long term prognoses remains uncertain, although it unequivocally increases return of spontaneous circulation. ${ }^{12}$ Recent randomised controlled trials showed slightly favourable (but non-significant) effects of pre-hospital adrenaline in improving long term survival, ${ }^{3}$ whereas observational studies have not indicated any significant favourable effects and some large scale registry based studies have even shown detrimental long term effects. ${ }^{5-10}$ 
These contradictory results could come from methodological shortcomings. The randomised controlled trials were underpowered. The observational studies could not adjust for time dependent imbalance of severity: timing of return of spontaneous circulation is a determinant of both outcome and treatment (those who rapidly respond to initial resuscitation procedures would have a higher chance of survival and a lower chance of being given of adrenaline). ${ }^{261112} \mathrm{~A}$ few registry based studies have focused on timing and shown favourable effects of early administration, but they did not adjust for the timing of return of spontaneous circulation. ${ }^{11}{ }^{13}$ In addition, previous studies ignored possible use of adrenaline in hospital; without this information, evaluation of pharmacological effects of adrenaline is impossible.

Evaluation of the effectiveness of the strategy of using pre-hospital adrenaline in improving long term outcomes in patients with out of hospital cardiac arrest would contribute to resolving the uncertainty about the role of adrenaline in pre-hospital resuscitation. ${ }^{2}$ We analysed a large scale national database in Japan, which provided sufficient power to detect any small effects, and used time dependent propensity score matching to adjust for the time dependent imbalance in patients inherent in observational studies.

\section{Methods \\ Study settings}

In Japan, the fire departments of municipal governments are responsible for emergency medical services, dispatching ambulances from fire stations. Each ambulance team consists of three emergency medical services personnel, usually including at least one emergency lifesaving technician trained to provide advanced life support (advanced airway management, placement of intravenous line, and defibrillation). Ambulances with doctors are also available but are not routinely used. ${ }^{14}$ Emergency lifesaving technicians certified after training in hospital are allowed to administer adrenaline (epinephrine) and perform endotracheal intubation with remote instruction from a doctor. Administration of adrenaline to patients with out of hospital cardiac arrest by emergency lifesaving technicians started in April 2006 with a small number of certified technicians, which gradually increased. Thus, during the study period Japanese emergency medical services consisted of a mixture of ambulance teams with and without the ability to administer pre-hospital adrenaline (a relatively small fraction of patients with out of hospital cardiac arrest received pre-hospital adrenaline), which allowed us to examine the effectiveness of pre-hospital adrenaline administered by emergency medical services. The emergency medical services resuscitation procedures follow Japanese guidelines for cardiopulmonary resuscitation, based on the American Heart Association guidelines current at that time. If patients with out of hospital cardiac arrest do not respond to initial cardiopulmonary resuscitation and defibrillation, a dose of $1 \mathrm{mg}$ intravenous adrenaline can be given under instruction from a doctor (other routes or other drugs are not permitted). Repeated doses can be given. Emergency medical services have no discretion to stop resuscitation at the scene; they are supposed to resuscitate and transport patients with out of hospital cardiac arrest to hospital if they are not definitely dead (in cases of decapitation and torso transection, for example). Details have been described elsewhere..$^{1011} 1415$

\section{Study design}

We used a controlled propensity matched retrospective cohort study to compare outcome in patients who received (adrenaline group) or did not receive (control group) pre-hospital adrenaline by emergency medical services. We used national registry data obtained from the All-Japan Utstein Registry of patients with out of hospital cardiac arrest. We restricted our comparison to pre-hospital use of adrenaline because the registry data did not include information on use in hospital. We could assume that almost all of the patients reaching the hospital without return of spontaneous circulation received adrenaline at the hospital. ${ }^{11} 16$ We adopted time dependent propensity score matching to assure comparability between adrenaline and control groups by balancing severity of the cardiac arrest and characteristics of the patients (see table $1 \Downarrow$ ) at each time interval (every minute from 6 to 30 minutes) from initiation of cardiopulmonary resuscitation by emergency medical services to administration of adrenaline..$^{17}$ All the analyses including propensity score calculation and outcome comparison were performed separately among those with an initial rhythm of ventricular fibrillation or pulseless ventricular tachycardia (VF/VT) and among those with non-VF/VT, because the initial rhythms definitively determine the cardiopulmonary resuscitation procedures and prognoses.

\section{Study participants}

We included patients aged 15-94 who had an out of hospital cardiac arrest witnessed by a bystander and were registered between January 2007 and December 2010 (figure $\Downarrow$ ). In some cases with no witness, cardiopulmonary resuscitation might have been not indicated but still carried out so we excluded them because emergency medical services have no discretion to terminate resuscitation. We also excluded patients who arrested after the arrival of emergency medical services (as we focused on cardiac arrest in situations without medical personnel), those who were given adrenaline after return of spontaneous circulation (re-arrest cases), those in whom arrest was attributable to external causes (such as trauma, drowning, poisoning, and asphyxia), and those with missing, contradictory, or outlying data (such as negative or long ( $>2$ hours) response interval). We also excluded those who were transported by ambulance without an emergency lifesaving technician or by ambulance with doctors.

\section{Data collection and variables}

Since 2005, the All-Japan Utstein Registry, a national registry managed by the Fire and Disaster Management Agency of Japan, has prospectively registered all patients with out of hospital cardiac arrest transported to hospital by emergency medical services in Japan. ${ }^{15}$ Emergency medical services staff collected data using an Utstein-style form, ${ }^{18}$ including age, sex, initial rhythm (VF/VT, pulseless electrical activity, asystole, and other), presumed cause of arrest, witnessed or not, description of bystander (family member, colleague, and other), bystander cardiopulmonary resuscitation including defibrillation with an automated external defibrillator, instruction in cardiopulmonary resuscitation to bystander by staff at emergency medical services control centre, activities of emergency medical services (defibrillation, airway management, intravenous line placement, administration of adrenaline), time course of activities (timing of the first dose of adrenaline was recorded; timings of additional doses, airway management, and placement of intravenous line were not recorded), and patient outcomes (spontaneous return of circulation before admission to hospital and long term survival). Failed attempts at airway management or intravenous line placement were not recorded. Information on treatment and return of spontaneous circulation after arrival at hospital was also not recorded. Intravenous line placement was not included 
in any analyses because it is a part of adrenaline administration procedures. Adrenaline doses were recorded but not included in the analyses because total doses were unknown without information on doses in hospital. If patients reached hospital without return of spontaneous circulation, control patients might have received adrenaline and patients in the adrenaline group might have received additional doses. ${ }^{116}$

The timings of activities of emergency medical services were measured with a clock synchronised within the emergency medical services system, recorded on the activity log forms, and then transcribed to the Utstein-style form. One of the three crew members took charge of data recording; they were trained to record and report the activities with the timing using the designated forms. The physician treating the patient at the hospital determined the cause of arrest clinically. Emergency medical services staff in charge of the data collection contacted the physician to seek information on survival and neurological function at one month or at discharge, whichever was earlier. If the patient survived, the physician assessed neurological function using the Glasgow-Pittsburgh cerebral performance category score: 1 (good), 2 (moderately disabled), 3 (severely disabled), 4 (vegetative state), or 5 (dead). ${ }^{18}$

The fire departments sent the data to the Fire and Disaster Management Agency, where they were compiled. ${ }^{14}$ The agency checked the data for missing or contradictory values and requested the fire departments to correct any errors detected to assure the data quality. Of the witnessed patients $(n=144074)$, $3636(2.5 \%)$ had missing timing data; of those otherwise eligible for the analyses ( $n=100677), 4598$ (4.6\%) had contradictory, outlying, or missing data in the variables that were used for propensity score calculation (figure $\Downarrow$ ).

In this study, patients administered adrenaline were defined as those with data on timing of administration; patients without such data were categorised as not receiving adrenaline. We assumed that this categorisation would underestimate rather than overestimate the effects of pre-hospital use of adrenaline and would lead to conservative results. Some patients who actually received adrenaline might have lacked data on timing.

\section{Time dependent propensity score matching}

Time dependent propensity score matching is a sequential risk set matching that uses time dependent propensity scores. We created pairs of adrenaline and control patients (1:1 matching) within a risk set at each time interval from the start of cardiopulmonary resuscitation by emergency medical services in a chronological order. ${ }^{17}{ }^{19}$ The risk set at time $t$ consisted of those who are at risk of treatment at time $t$, excluding those who have attained return of spontaneous circulation (no indication of treatment by emergency medical services) or reached hospital (no possibility of treatment by emergency medical services) by time $t$. The sequential matching should not depend on future information to avoid selection biases. Therefore, patient who later received adrenaline as well as those who never received it were included in the risk set and could be selected as controls (a control group consisting of patients who never received adrenaline alone would accumulate those with favourable outcomes because patients who were slow to attain return of spontaneous circulation might have received adrenaline whereas those who attained return of spontaneous circulation relatively early would not). ${ }^{17} 19$

The time dependent propensity scores were predicted values, denoted by $S$, in the following Cox proportional hazard model:

$\mathrm{h}(\mathrm{t})=\mathrm{h}_{0}(\mathrm{t}) \exp \left(\mathrm{b}_{1} \mathrm{x}_{1}+\mathrm{b}_{2} \mathrm{x}_{2}+\mathrm{b}_{3} \mathrm{x}_{3}+\ldots+\mathrm{b}_{\mathrm{p}} \mathrm{x}_{\mathrm{p}}\right)$, where $\mathrm{h}(\mathrm{t})$ and $\mathrm{h}_{0}(\mathrm{t})$ are the hazard function and corresponding baseline hazard function, respectively, which are both constants for given $\mathrm{t}$; $\mathrm{t}$ was the time interval from the start of cardiopulmonary resuscitation by emergency medical services to administration of adrenaline by emergency medical services; and $b_{1}, b_{2}, b_{3}, \ldots b_{p}$ and $x_{1}, x_{2}, x_{3}, \ldots x_{p}$ were estimated coefficients and covariates, respectively.

The model included all the variables obtainable in the field as predictors, except for the description of the bystander (family member or not), which violated the proportionality assumption, and the time from the onset of cardiopulmonary resuscitation by emergency medical services to defibrillation, which caused multicollinearity. The predictors, categorised in table $1 \Downarrow$, were year, sex, age, bystander cardiopulmonary resuscitation, bystander defibrillation, cardiopulmonary resuscitation instruction given, initial rhythm, time interval from call to emergency medical services to patient contact, number of attempts at defibrillation by emergency medical services, and advanced airway management. The model also included prefecture categories according to the proportions of patients with out-of-hospital cardiac arrest witnessed by a bystander who received adrenaline by emergency medical services (four categories: $<5 \%, 5-<15 \%, 15-<30 \%$, and $\geq 30 \%$ ) to adjust for regionally different environments influencing pre-hospital use of adrenaline.

We created pairs having the closest propensity scores within a calliper (in each pair, the difference in the propensity scores should be within this predetermined width), a quarter of the standard deviation of the propensity scores, without replacement, using a user developed psmatch 2 program in $\mathrm{Stata}^{20}{ }^{20}$ as described by Guo and Fraser. ${ }^{21}$ In the matching process, the timing of administration of adrenaline from the start of cardiopulmonary resuscitation by emergency medical services for the matching was restricted from $t=6$ to $t=30$ (minutes); administration at $t<6$ was considered to be technically difficult and administration at $t>30$ was rare. The matched pairs were removed from the subsequent procedures. The matching created 1990 pairs for VT/VF and 9058 pairs for non-VF/VT patients.

We showed the imbalance between groups of patients using differences of proportions as all variables were categorical (appendix 1). We used imbalance proportion defined as the sum of absolute differences of proportions between groups among all levels (categories) in each variable and using standardised difference of proportions defined as

$\sqrt{ }\left(\mathrm{P}_{1}-\mathrm{P}_{2}\right)^{\prime} \Sigma^{-1}\left(\mathrm{P}_{1}-\mathrm{P}_{2}\right)$

where a $(k-1)$ dimensional proportion vector $\mathrm{P}_{1}-\mathrm{P}_{2}$ in a variable with $\mathrm{k}$ levels is considered to approximately follow $(\mathrm{k}-1)$ dimensional normal distribution

$\mathrm{N}_{\mathrm{k}-1}(0, \Sigma)$

Here, $\mathrm{P}_{1}$ and $\mathrm{P}_{2}$ are $(\mathrm{k}-1)$ dimensional proportion vectors of a variable with $\mathrm{k}$ levels in VF/VT and non-VF/VT groups, respectively. $\sum$ is a $(\mathrm{k}-1)$ dimensional diagonal matrix with the (i,i) element being variance of the $\mathrm{i}$-th element $(\mathrm{i}=1,2, \ldots, \mathrm{k}-1)$ of the proportion vector $\mathrm{P}_{1}-\mathrm{P}_{2}$.

\section{Endpoints}

The endpoints were overall survival and neurologically intact survival with the Glasgow-Pittsburgh cerebral performance category score 1-2 at one month or at discharge, whichever was earlier. Most of the previous studies used survival at one month or at discharge with and without neurological outcomes, which allowed us to compare our findings with the previous ones. 


\section{Statistical analysis}

After describing the participants' characteristics (before and after matching), we applied conditional logistic regression models to the matched pairs, with and without adjustment for the variables that were not included in the development of the propensity score, to determine the relation between

administration of adrenaline and the endpoints. As the outcomes were measured only once at a fixed time point (at one month or discharge), we did not use a Cox proportional hazard model for the outcome comparisons. The covariates for adjustment in the logistic regression models were bystander description, time from the onset of cardiopulmonary resuscitation by emergency medical services to hospital arrival, time from the onset of cardiopulmonary resuscitation by emergency medical services to the first defibrillation, and presumed cause of arrest. As the sample sizes were determined by the number of patients registered in the database, we calculated the power of the Wald tests of the parameters being equal to 0 (null hypothesis) under a significance level of $5 \%$ (two sided). ${ }^{22}$ All analyses were performed with Stata version 12.1 (StataCorp, College Station, TX, USA).

\section{Results}

\section{Patients' characteristics}

Of the 438005 patients aged 15-94 with out of hospital cardiac arrest registered in the database during the study period, 96079 met the inclusion criteria (figure $\Downarrow$ ). Pre-hospital adrenaline (epinephrine) was administered in $16.5 \%$ of those with VF/VT (14 943) and $13.5 \%$ of those with non-VF/VT (81 136). The proportions of overall survival and neurologically intact survival at one month or at discharge were $7.7 \%$ and $3.9 \%$, respectively, among all patients; $26.8 \%$ and $17.6 \%$, respectively, among those with VF/VT; and $4.2 \%$ and $1.3 \%$, respectively, among those with non-VF/VT.

Among all patients, administration of adrenaline increased year by year during the study period regardless of the initial rhythm (table $1 \Downarrow$ ). Those who received adrenaline tended to have received cardiopulmonary resuscitation from a bystander after instruction, had advanced airway management, had a long interval from call to emergency medical services to patient contact, and had multiple defibrillations, delayed defibrillation, delayed hospital arrival, and delayed return of spontaneous circulation.

The propensity score matching balanced the distribution of each variable included in the propensity score calculation, with the imbalance proportions varying from $0.1-50.5 \%$ in VF/VT and $0.4-50.9 \%$ in non-VF/VT patients before matching and decreasing to $0.10-6.6 \%$ and $0.04-3.4 \%$, respectively, after matching; the standardised differences between groups decreasing from 0.02-16.9 in VF/VT and 0.21-34.8 in non-VF/VT patients to $0.02-2.63$ and $0.02-3.98$, respectively (tables 1 and $2 \Downarrow$ ).

\section{Main results}

In the matched samples, the adrenaline treated groups showed higher proportions of overall survival at one month or at discharge than the control groups in both VF/VT $(17.0 \% v$ $13.4 \%, 3.6$ percentage points difference) and non-VF/VT patients $(4.0 \% \vee 2.4 \%, 1.6$ percentage points difference) (table $2 \Downarrow)$. The proportion of patients with neurologically intact survival did not differ between adrenaline and control groups in VF/VT patients $(6.6 \% v 6.6 \%)$; it was higher in the adrenaline group among non-VF/VT patients $(0.7 \% v 0.4 \%, 0.2$ percentage point difference).

For VF/VT patients, overall survival was significantly associated with administration of adrenaline (unadjusted odds ratio 1.34 (95\% confidence interval 1.12 to 1.60 ); adjusted odds ratio 1.36 (1.13 to 1.63$)$ ); whereas neurologically intact survival was not associated with use of adrenaline (unadjusted odds ratio 1.01, 0.78 to 1.30 ; adjusted odds ratio $1.02,0.78$ to 1.33 ) (table $3 \Downarrow$ ). For non-VF/VT patients, overall survival was also significantly associated with use of adrenaline (unadjusted odds ratio 1.72 (1.45 to 2.04), adjusted odds ratio 1.78 (1.49 to 2.13)). For neurologically intact survival, the unadjusted odds ratio was significant at 1.57 (1.04 to 2.37), but the adjusted odds ratio was not significant $(1.55,0.99$ to 2.41$)$. The powers of the Wald tests exceeded $80 \%$ for the analyses of overall survival, and equalled $50 \%$ for that of neurologically intact survival among those with non-VF/VT.

\section{Discussion}

Patients who have a cardiac arrest out of hospital and who are given adrenaline (epinephrine) by emergency medical services have more favourable long term outcomes than those not given adrenaline. With adjustment for the time dependent patient imbalance, the present study resolves the uncertainty about the role of pre-hospital adrenaline. Though the overall proportion of patients who survived was higher in the adrenaline group than in the control group, however, neurologically intact survival was similar.

\section{Strengths and limitations}

Our study was sufficiently powered and successfully adjusted for time dependent severity of cardiac arrest, using sequential risk set matching with time dependent propensity scores (in other words, it adjusted for the varying duration of resuscitation procedures). ${ }^{2}$ This way, for those who received adrenaline at a certain time point, their controls were selected from those who were, at that time point, still at risk for adrenaline use and had similar propensity scores (similar likelihood of receiving adrenaline). ${ }^{17}{ }^{19}$ Some variables used in calculating propensity scores (such as age, time before arrival of emergency medical services, rhythms) were also related to the likelihood of being successfully resuscitated, resulting in somewhat similar likelihood of successful resuscitation between the two groups as far as the involved variables were concerned. Without this adjustment, severely affected patients who required longer resuscitation procedures would tend to accumulate in the group treated with adrenaline.

Several limitations should be noted. Firstly, the database did not include information on the treatment in hospital. Without knowing the in hospital and total doses of adrenaline, we could not evaluate the pharmacological effects of adrenaline but could compare only the strategies with and without pre-hospital adrenaline using information on the timing of the first pre-hospital doses. Nevertheless, in terms of the survival outcomes, the timing of the first dose matters ${ }^{11}{ }^{13}$ but the total of the doses might not. ${ }^{23}$ Furthermore, as we did not have information on care after resuscitation, which obviously influences the outcomes, ${ }^{24}$ we could not adjust for confounding from differences between hospitals; however, we believe the confounding effects were minimal because hospital abilities are unlikely to influence the decision to use pre-hospital adrenaline. ${ }^{11}$ Secondly, the database did not record information on treatment decisions (when, how, and why what decisions were made) or failed attempts at treatment but recorded only actual treatment. 
Some treatment decisions to give adrenaline might not have been executed because of return of spontaneous circulation before the treatment or failed placement of an intravenous line. Discrepancies between the decisions and the actual treatment might have caused some biases, although we tried to obtain the best estimate of the effects of adrenaline by fully using available information.

Thirdly, unmeasured factors that influence both treatment decisions and patient outcomes might have confounded the results; the magnitude of their influences could not be quantified. For example, short distance to the hospital could be a determinant of both good outcomes and quick transfer of patients without pre-hospital adrenaline ${ }^{25}$; existence of comorbidities and their severities might also have influenced both the decisions and the outcomes.

Fourthly, improved overall survival might have resulted from improved performance of resuscitation by emergency lifesaving technicians certified for adrenaline administration because they had additional training. We could not adjust for the skills of emergency lifesaving technicians as we had no information on certification status and quality of resuscitation. Records of these factors (in hospital treatment, treatment decision, failed attempts at treatment, distance to hospital, and certification status) would allow us to reduce confounding and achieve better balance.

\section{Comparison with other studies}

Our findings contradict the harmful long term effects of adrenaline shown in previous observational studies, including a recent Japanese study that used the same database. ${ }^{9}{ }^{10}$ Hagihara and colleagues used propensity score matching and logistic regression to adjust for confounding and showed that pre-hospital administration of adrenaline might reduce the chances of overall and neurologically intact survival at one month by about $50 \%$ and $80 \%$, respectively. ${ }^{10}$ Their statistical models including only time independent covariates, however, could not appropriately adjust for time dependent imbalance.

Other studies using large scale registry data in Japan were able to partially address the time dependent imbalance. Hayashi and colleagues ${ }^{13}$ using a local registry (Osaka Utstein registry) and Nakahara and colleagues ${ }^{11}$ using the national registry adjusted for the timing of administration of adrenaline in a manner similar to the present study but somewhat imprecisely. Both showed favourable effects of early administration. They could not, however, adjust for the timing of return of spontaneous circulation and hospital arrival among the control group, important determinants of both adrenaline administration and outcomes, for which we fully adjusted on a minute by minute basis. Our findings could be explained by the timing of treatment: administration by emergency medical services minimises the interval before the patients receives adrenaline.

The contrasting findings with the same data result from different analytical methods of adjustment for important confounding. Similarly contrasting findings resulted from the same randomised controlled trial data in Norway that used different analytical methods: per-protocol analysis showed that actual adrenaline use was associated with decreased survival to discharge contrary to the original results with intention to treat analysis. ${ }^{32}$ Categorisation of patients based on actual treatment caused imbalance of severity of the cardiac arrest: less severely affected patients tended to attain return of spontaneous circulation before adrenaline administration in the intervention group.

\section{Meaning of the study}

The present findings that pre-hospital administration of adrenaline by emergency medical services resulted in favourable survival outcomes do not simply support the expansion of such strategies. Strategies of pre-hospital adrenaline given by emergency medical services might mainly increase survival with severe neurological disabilities among those with VF/VT and minimally increase neurologically intact survival among those with non-VF/VT. To determine the role of routine use of pre-hospital adrenaline, further studies are required to evaluate whether adrenaline really disproportionately increases disabled survival, and societal debate would then be required on the issue of such a potential increase of severely disabled survival.

In determining the role of adrenaline, we also have to consider the effects of post-resuscitation care such as hypothermia, which has been shown to improve long term neurological outcomes. ${ }^{24} 26-28$ Improved post-resuscitation care might have the potential to modify the effects of adrenaline and to increase neurologically intact survival among those with VF/VT after administration. Increasing overall survival is the prerequisite of increasing neurologically intact survival.

The minimal absolute difference of neurologically intact survival between adrenaline and control groups ( 0.3 percentage point) among those with non-VF/VT reflected the extremely poor prognoses of these patients. Although the small absolute difference suggests a minimal impact of pre-hospital use of adrenaline in the population, adrenaline might be considered as the last resort after other interventions fail if there is the slightest possibility of increasing survival.

Showing the effectiveness of strategies that use pre-hospital adrenaline in improving outcomes of patients with out of hospital cardiac arrest, despite continued uncertainty about whether the improvement resulted from pre-hospital adrenaline use or additional training to emergency lifesaving technicians, would contribute to consolidating the weakest link of the "chain of survival," the fourth link: early access to advanced life support including drug use..$^{29}$ Our findings provide evidence supporting the benefit of streamlining the fourth link; this would ease the dilemma that emergency lifesaving technicians have faced ("What if our treatment is doing some harm to the patient?") as there had been no such evidence up to the present.

\section{Generalisability}

The present results might not generalise to other countries, although the generalisability to the whole population of patients with out of hospital cardiac arrest witnessed by a bystander in Japan can be guaranteed as all such patients are registered in the national registry. Pre-hospital care systems, including termination rules and hospital capacities for post-arrest care, vary from country to country, which could modify the effects of adrenaline. Our findings cannot be generalised to cases with no witness or witnessed by emergency medical services; such cases were excluded from our analyses. Therefore, previously and newly collected data in other countries should be analysed to appropriately handle time dependent patient imbalance.

\section{Conclusions}

Our study showed some favourable effects of pre-hospital administration of adrenaline by emergency medical services on long term outcomes of patients with out of hospital cardiac arrest after we accounted for time dependent patient imbalances, which previous observational studies had not fully addressed. The absolute increase of neurologically intact survival, however, was minimal. Previous observational data could be reanalysed 
with the methods we used. The registry of out of hospital cardiac arrest should collect more information (such as comorbidities, post-resuscitation care, abilities of emergency lifesaving technician, and hospital treatment) to more accurately evaluate the effectiveness of pre-hospital use of adrenaline. Furthermore, to determine the efficacy of adrenaline in resuscitation, large scale randomised controlled trials might be needed.

Part of this study was presented at the Kanto regional meeting of Japanese Association for Acute Medicine, in Tokyo, Japan, 28 February 2013.

Contributors: All authors contributed to the study design; SN and TS obtained the data; SN, JT, HT, and Ml analysed the data; SN drafted the manuscript; JT, HT, MI, MN, NM, and TS contributed to interpretation and manuscript revision. All authors approved the manuscript. SN is guarantor.

Funding: This research received no specific grant from any funding agency in the public, commercial, or not-for-profit sectors.

Competing interests: All authors have completed the ICMJE uniform disclosure form at www.icmje.org/coi_disclosure.pdf and declare: no support from any organisation for the submitted work; no financial relationships with any organisations that might have an interest in the submitted work in the previous three years; no other relationships or activities that could appear to have influenced the submitted work.

Ethical approval: The institutional review board of St Marianna University approved the study protocol and waived the requirement for informed consent (ID 1714).

Data sharing: Statistical codes are available from the corresponding author. The data used in this study are public domain data available from the Fire and Disaster Management Agency. No additional data are available.

Transparency statement: SN affirms that the manuscript is an honest, accurate, and transparent account of the study being reported; that no important aspects of the study have been omitted; and that any discrepancies from the study as planned (and, if relevant, registered) have been explained.

1 Morrison LJ, Deakin CD, Morley PT, Callaway CW, Kerber RE, Kronick SL, et al. Part 8 : Advanced life support: 2010 International Consensus on Cardiopulmonary Resuscitation and Emergency Cardiovascular Care Science With Treatment Recommendations. Circulation 2010;122:S345-421.

2 McQueen C, Gates S, Perkins GD. Adrenaline for the pharmacological treatment of cardiac arrest... going, going, gone? Resuscitation 2012;83:921-2.

3 Olasveengen TM, Sunde K, Brunborg C, Thowsen J, Steen PA, Wik L. Intravenous drug administration during out-of-hospital cardiac arrest: a randomized trial. JAMA 2009;302:2222-9.

4 Jacobs IG, Finn JC, Jelinek GA, Oxer HF, Thompson PL. Effect of adrenaline on survival in out-of-hospital cardiac arrest: a randomised double-blind placebo-controlled trial. Resuscitation 2011;82:1138-43.

5 Bur A, Kittler H, Sterz F, Holzer M, Eisenburger P, Oschatz E, et al. Effects of bystander first aid, defibrillation and advanced life support on neurologic outcome and hospital costs in patients after ventricular fibrillation cardiac arrest. Intensive Care Med 2001;27:1474-80.

6 Herlitz J, Ekstrom L, Wennerblom B, Axelsson A, Bang A, Holmberg S. Adrenaline in out-of-hospital ventricular fibrillation. Does it make any difference? Resuscitation 1995;29:195-201.

7 Ong ME, Tan EH, Ng FS, Panchalingham A, Lim SH, Manning PG, et al. Survival outcomes with the introduction of intravenous epinephrine in the management of out-of-hospital cardiac arrest. Ann Emerg Med 2007;50:635-42.
8 Stiell IG, Wells GA, Field B, Spaite DW, Nesbitt LP, De Maio VJ, et al. Advanced cardiac life support in out-of-hospital cardiac arrest. N Engl J Med 2004;351:647-56.

9 Holmberg M, Holmberg S, Herlitz J. Low chance of survival among patients requiring adrenaline (epinephrine) or intubation after out-of-hospital cardiac arrest in Sweden. Resuscitation 2002;54:37-45.

10 Hagihara A, Hasegawa M, Abe T, Nagata T, Wakata Y, Miyazaki S. Prehospital epinephrine use and survival among patients with out-of-hospital cardiac arrest. JAMA 2012;307:1161-8.

11 Nakahara S, Tomio J, Nishida M, Morimura N, Ichikawa M, Sakamoto T. Association between timing of epinephrine administration and intact neurologic survival following out-of-hospital cardiac arrest in Japan: a population-based prospective observational study. Acad Emerg Med 2012;19:782-92.

12 Olasveengen TM, Wik L, Sunde K, Steen PA. Outcome when adrenaline (epinephrine) was actually given vs. not give - post hoc analysis of a randomized clinical trial. Resuscitation 2012;83:327-32.

13 Hayashi Y, Iwami T, Kitamura T, Nishiuchi T, Kajino K, Sakai T, et al. Impact of early intravenous epinephrine administration on outcomes following out-of-hospital cardiac arrest. Circ J 2012;76:1639-45.

14 Tanigawa K, Tanaka K. Emergency medical service systems in Japan: past, present, and future. Resuscitation 2006:69:365-70.

15 Kitamura T, Iwami T, Kawamura T, Nagao K, Tanaka H, Hiraide A. Nationwide public-access defibrillation in Japan. N Engl J Med 2010;362:994-1004.

16 SOS-KANTO study group. Cardiopulmonary resuscitation by bystanders with ches compression only (SOS-KANTO): an observational study. Lancet 2007;369:920-6.

17 Lu B. Propensity score matching with time-dependent covariates. Biometrics 2005;61:721-8.

18 Jacobs I, Nadkarni V, Bahr J, Berg RA, Billi JE, Bossaert L, et al. Cardiac arrest and cardiopulmonary resuscitation outcome reports: update and simplification of the Utstein templates for resuscitation registries: a statement for healthcare professionals from a task force of the International Liaison Committee on Resuscitation (American Heart Association, European Resuscitation Council, Australian Resuscitation Council, New Zealand Resuscitation Council, Heart and Stroke Foundation of Canada, InterAmerican Heart Foundation, Resuscitation Councils of Southern Africa). Circulation 2004:110:3385-97.

19 Li Y, Propert K, Rosenbaum P. Balanced risk set matching. JAMA 2001;96:870-82.

20 Leuven E, Sianesi B. PSMATCH2: Stata module to perform full Mahalanobis and propensity score matching, common support graphing, and covariate imbalance testing. http://ideas. repec.org/c/boc/bocode/s432001.html.

21 Guo S, Fraser MW. Propensity score matching and related models. In: Guo S, Fraser MW. Propensity score analysis: statistical methods and applications. SAGE, 2010:127-210.

22 Hosmer DW, Lemeshow S. Sample size issues when fitting logistic regression models. In: Applied logistic regression. Wiley-International Publication, 2000:339-47.

23 Vandycke $C$, Martens P. High dose versus standard dose epinephrine in cardiac arrest - a meta-analysis. Resuscitation 2000;45:161-6.

24 Peberdy MA, Callaway CW, Neumar RW, Geocadin RG, Zimmerman JL, Donnino M, et al. Part 9: post-cardiac arrest care: 2010 American Heart Association Guidelines for Cardiopulmonary Resuscitation and Emergency Cardiovascular Care. Circulation 2010;122:S768-86

25 Shirako T, Noguchi H, Araki T, , Ueyama M, Kitagawa Y, Takeuchi A, et al. Status analysis of pre-hospital intravenous adrenaline administration in the management of out-of-hospital cardio-pulmonary arrest in Nagoya. J Jpn Soc Emerg Med 2010;13:341-8.

26 Bernard SA, Gray TW, Buist MD, Jones BM, Silvester W, Gutteridge G, et al. Treatment of comatose survivors of out-of-hospital cardiac arrest with induced hypothermia. N Eng $J$ Med 2002;346:557-63.

27 Hypothermia After Cardiac Arrest Group. Mild therapeutic hypothermia to improve the neurologic outcome after cardiac arrest. N Engl J Med 2002;346:549-56.

28 Tagami T, Hirata K, Takeshige T, Matsui J, Takinami M, Satake M, et al. Implementation of the fifth link of the chain of survival concept for out-of-hospital cardiac arrest. Circulation 2012:126:589-97.

29 Berg RA, Hemphill R, Abella BS, Aufderheide TP, Cave DM, Hazinski MF, et al. Part 5: adult basic life support: 2010 American Heart Association Guidelines for Cardiopulmonary Resuscitation and Emergency Cardiovascular Care. Circulation 2010;122(18 suppl 3):S685-705.

Accepted: 10 November 2013

\section{Cite this as: BMJ 2013;347:f6829}

This is an Open Access article distributed in accordance with the Creative Commons Attribution Non Commercial (CC BY-NC 3.0) license, which permits others to distribute, remix, adapt, build upon this work non-commercially, and license their derivative works on different terms, provided the original work is properly cited and the use is non-commercial. See: http://creativecommons.org/licenses/by-nc/3.0/. 


\title{
What is already known on this topic
}

Early access to emergency medical care, cardiopulmonary resuscitation, and defibrillation have clear benefit on survival after out of hospital cardiac arrest

Whether pre-hospital use of adrenaline improves long term prognoses after out of hospital cardiac arrest remains uncertain, although it unequivocally increases return of spontaneous circulation

Some observational studies have shown harmful effects of pre-hospital adrenaline, whereas recent randomised controlled trials have shown slightly favourable (non-significant) effects

\section{What this study adds}

There were some favourable long term outcomes among patients with out of hospital cardiac arrest who received pre-hospital adrenaline from emergency medical services

The overall survival proportion was higher among those who received adrenaline than those who did not, but neurologically intact survival was similar

This study accounted for time dependent patient imbalance, which previous observational studies had not done

\section{Tables}

\begin{abstract}
Table 1/ Characteristics, outcomes, and imbalance indicators of patients with witnessed out of hospital cardiac arrest by initial rhythms and pre-hospital administration of adrenaline (epinephrine) before propensity score matching. Figures are numbers (percentages) unless stated otherwise
\end{abstract}

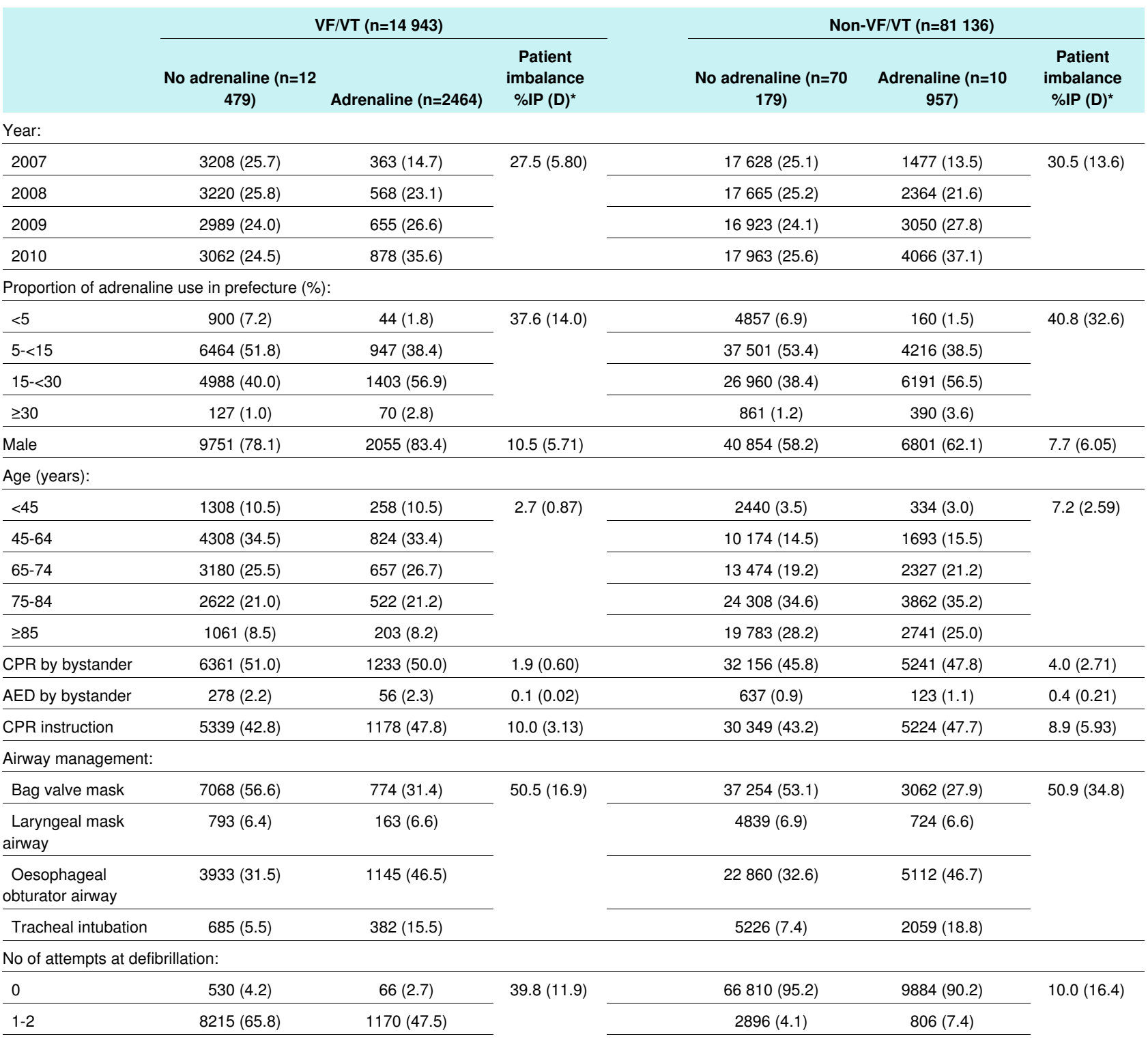


Table 1 (continued)

\begin{tabular}{|c|c|c|c|c|c|c|}
\hline & \multicolumn{3}{|c|}{ VF/VT (n=14 943) } & \multicolumn{3}{|c|}{ Non-VF/VT ( $n=81$ 136) } \\
\hline & $\begin{array}{l}\text { No adrenaline }(n=12 \\
479)\end{array}$ & Adrenaline $(n=2464)$ & $\begin{array}{l}\text { Patient } \\
\text { imbalance } \\
\% \text { IP (D) }{ }^{*}\end{array}$ & $\begin{array}{l}\text { No adrenaline }(n=70 \\
179)\end{array}$ & $\begin{array}{c}\text { Adrenaline }(\mathrm{n}=10 \\
957)\end{array}$ & $\begin{array}{c}\text { Patient } \\
\text { imbalance } \\
\% \mathrm{IP}(\mathrm{D})^{*}\end{array}$ \\
\hline$\geq 3$ & $3734(29.9)$ & $1228(49.8)$ & & $473(0.7)$ & $267(2.4)$ & \\
\hline \multicolumn{7}{|c|}{ EMS call-contact interval (min): } \\
\hline$\leq 6$ & $4531(36.3)$ & $673(27.3)$ & \multirow[t]{4}{*}{$18.0(5.14)$} & $19125(27.3)$ & $2421(22.1)$ & \multirow[t]{4}{*}{$13.2(6.33)$} \\
\hline $7-8$ & $3743(30.0)$ & $796(32.3)$ & & $20452(29.1)$ & $3036(27.7)$ & \\
\hline $9-10$ & $2304(18.5)$ & $513(20.8)$ & & $14456(20.6)$ & $2473(22.6)$ & \\
\hline$\geq 11$ & $1901(15.2)$ & $482(19.6)$ & & $16146(23.0)$ & $3027(27.6)$ & \\
\hline $\begin{array}{l}\text { Arrest had cardiac } \\
\text { origin }\end{array}$ & $11283(90.4)$ & $2288(92.9)$ & - & $43643(62.2)$ & $7431(67.8)$ & - \\
\hline $\begin{array}{l}\text { Family member was } \\
\text { bystander }\end{array}$ & $7573(60.7)$ & $1542(62.6)$ & - & $48888+(69.7)$ & $7661(69.9)$ & - \\
\hline \multicolumn{7}{|c|}{ CPR (EMS)-defibrillation interval (min): } \\
\hline No defibrillation & $530(4.2)$ & $66(2.7)$ & - & $66810(95.2)$ & $9884(90.2)$ & - \\
\hline $1-2$ & $7389(59.2)$ & $1480(60.1)$ & - & $322(0.5)$ & $49(0.4)$ & - \\
\hline $3-4$ & $3330(26.7)$ & $670(27.2)$ & - & $402(0.6)$ & $85(0.8)$ & - \\
\hline$\geq 5$ & $1230(9.9)$ & $248(10.1)$ & - & $2645(3.8)$ & $938 \ddagger(8.6)$ & - \\
\hline \multicolumn{7}{|c|}{ CPR (EMS)-hospital arrival interval (min): } \\
\hline$\leq 17$ & $4182(33.5)$ & $221(9.0)$ & - & $22259(31.7)$ & $910(8.3)$ & - \\
\hline $18-22$ & $3246(26.0)$ & $502(20.4)$ & - & $17523(25.0)$ & $2149(19.6)$ & - \\
\hline $23-29$ & $2900(23.2)$ & $821(33.3)$ & - & $16974(24.2)$ & 3565 (32.5) & - \\
\hline$\geq 30$ & $2151(17.2)$ & $920(37.3)$ & - & $13423(19.1)$ & $4333(39.5)$ & - \\
\hline \multicolumn{7}{|c|}{ CPR (EMS)-ROSC interval (min): } \\
\hline No ROSC & $8975(71.9)$ & $1932(78.4)$ & - & $66194(94.3)$ & $8929(81.5)$ & - \\
\hline$\leq 6$ & $1596(12.8)$ & $10(0.4)$ & - & $1800(2.6)$ & $12(0.1)$ & - \\
\hline 7-12 & $1228(9.8)$ & $102(4.1)$ & - & $1144(1.6)$ & $288(2.6)$ & - \\
\hline $13-21$ & $566(4.5)$ & $268(10.9)$ & - & $760(1.1)$ & $947(8.6)$ & - \\
\hline$\geq 22$ & $114(0.9)$ & $152(6.2)$ & - & $281(0.4)$ & $781(7.1)$ & - \\
\hline \multicolumn{7}{|l|}{ Outcomes: } \\
\hline Overall survival & $3594(28.8)$ & $406(16.5)$ & - & $2963(4.2)$ & $428 \S(3.9)$ & - \\
\hline $\begin{array}{l}\text { Neurologically intact } \\
\text { survival (CPC 1-2) }\end{array}$ & 2466 (19.8) & $169(6.9)$ & - & $1023(1.5)$ & $67(0.6)$ & - \\
\hline
\end{tabular}

VFNT=ventricular fibrillation or ventricular tachycardia; $E M S=$ =emergency medical service; $C P R=$ cardiopulmonary resuscitation; $A E D=$ automated external defibrillator; ROSC=return of spontaneous circulation; CPC=Glasgow-Pittsburgh cerebral performance category score.

${ }^{*}$ Variables included in propensity score development show imbalance indicators. IP denotes imbalance proportion defined by sum of absolute differences of proportions between groups among all levels in each variable. $D$ denotes standardised difference defined as $\sqrt{ }\left(P_{1}-P_{2}\right)^{\prime} \Sigma^{-1}\left(P_{1}-P_{2}\right)$, where $(k-1)$ dimensional proportion vector $P_{1}-P_{2}$ in variable with $k$ levels is considered to follow $(k-1)$ dimensional normal distribution $N_{k-1}(0, \Sigma)$. Here, $P 1$ and $P 2$ are $(k-1)$ dimensional proportion vectors of variable with $k$ levels in VF/VT and non-VF/VT groups, respectively. $\Sigma$ is $(k-1)$ dimensional diagonal matrix with (i,i) element being variance of $i$-th element $(\mathrm{i}=1,2, \ldots, \mathrm{k}-1)$ of proportion vector $\mathrm{P}_{1}-\mathrm{P}_{2}$.

†Data missing in two patients.

fData missing in one patient.

$\S$ Data missing in one patient. 
Table 2/ Characteristics, outcomes, and imbalance indicators of patients with witnessed out of hospital cardiac arrest by initial rhythms and pre-hospital administration of adrenaline (epinephrine) after propensity score matching. Figures are numbers (percentages) unless stated otherwise

\begin{tabular}{|c|c|c|c|c|c|c|}
\hline & \multicolumn{3}{|c|}{ VF/VT (n=3980) } & \multicolumn{3}{|c|}{ Non-VF/VT ( $n=18$ 116) } \\
\hline & $\begin{array}{l}\text { No adrenaline } \\
(n=1990)\end{array}$ & Adrenaline ( $n=1990)$ & $\begin{array}{l}\text { Patient } \\
\text { imbalance } \\
\% \text { IP (D)* }\end{array}$ & $\begin{array}{l}\text { No adrenaline } \\
(n=9058)\end{array}$ & Adrenaline (n=9058) & $\begin{array}{c}\text { Patient } \\
\text { imbalance \%IP } \\
\text { (D) }\end{array}$ \\
\hline \multicolumn{7}{|l|}{ Year: } \\
\hline 2007 & $312(15.7)$ & $305(15.3)$ & \multirow[t]{4}{*}{$2.4(0.39)$} & 1216 (13.4) & $1271(14.0)$ & \multirow[t]{4}{*}{$1.5(0.45)$} \\
\hline 2008 & $480(24.1)$ & $463(23.3)$ & & $1999(22.1)$ & $1995(22.0)$ & \\
\hline 2009 & $509(25.6)$ & $520(26.1)$ & & $2509(27.7)$ & $2520(27.8)$ & \\
\hline 2010 & $689(34.6)$ & $702(35.3)$ & & $3334(36.8)$ & $3272(36.1)$ & \\
\hline \multicolumn{7}{|c|}{ Proportion of adrenaline use in prefecture (\%): } \\
\hline$<5$ & $29(1.5)$ & $43(2.2)$ & \multirow[t]{4}{*}{$3.4(0.77)$} & $119(1.3)$ & $146(1.6)$ & \multirow[t]{4}{*}{$1.8(0.93)$} \\
\hline $5-<15$ & $817(41.1)$ & $814(40.9)$ & & $3606(39.8)$ & $3625(40.0)$ & \\
\hline $15-<30$ & $1108(55.7)$ & $1077(54.1)$ & & $5081(56.1)$ & $5001(55.2)$ & \\
\hline$\geq 30$ & $36(1.8)$ & $56(2.8)$ & & $252(2.8)$ & $286(3.2)$ & \\
\hline Male & $1708(85.8)$ & $1642(82.5)$ & $6.6(2.63)$ & $5600(61.8)$ & $5622(62.1)$ & $0.5(0.26)$ \\
\hline \multicolumn{7}{|l|}{ Age (years): } \\
\hline$<45$ & $200(10.1)$ & $200(10.1)$ & \multirow[t]{5}{*}{$4.0(0.65)$} & $243(2.7)$ & $278(3.1)$ & \multirow[t]{5}{*}{$1.2(0.37)$} \\
\hline $45-64$ & $695(34.9)$ & $674(33.9)$ & & $1379(15.2)$ & $1397(15.4)$ & \\
\hline $65-74$ & $545(27.4)$ & $526(26.4)$ & & $1929(21.3)$ & $1908(21.1)$ & \\
\hline $75-84$ & $402(20.2)$ & $422(21.2)$ & & $3217(35.5)$ & $3204(35.4)$ & \\
\hline$\geq 85$ & $148(7.4)$ & $168(8.4)$ & & $2290(25.3)$ & $2271(25.1)$ & \\
\hline CPR by bystander & $984(49.4)$ & 985 (49.5) & $0.1(0.02)$ & $4275(47.2)$ & $4273(47.2)$ & $0.04(0.02)$ \\
\hline AED by bystander & $28(1.4)$ & $45(2.3)$ & $1.7(0.27)$ & $75(0.8)$ & $105(1.2)$ & $0.7(0.22)$ \\
\hline CPR instruction & $943(47.4)$ & 927 (46.6) & $1.6(0.35)$ & $4310(47.6)$ & $4288(47.3)$ & $0.5(0.23)$ \\
\hline \multicolumn{7}{|l|}{ Airway management: } \\
\hline Bag valve mask & $704(35.4)$ & 663 (33.3) & \multirow[t]{4}{*}{$4.6(0.89)$} & $2676(29.5)$ & $2695(29.8)$ & \multirow[t]{4}{*}{$1.4(0.61)$} \\
\hline $\begin{array}{l}\text { Laryngeal mask } \\
\text { airway }\end{array}$ & $114(5.7)$ & $137(6.9)$ & & $572(6.3)$ & $616(6.8)$ & \\
\hline $\begin{array}{c}\text { Oesophageal } \\
\text { obturator airway }\end{array}$ & $909(45.7)$ & $904(45.4)$ & & $4229(46.7)$ & $4182(46.2)$ & \\
\hline Tracheal intubation & $263(13.2)$ & $286(14.4)$ & & $1581(17.5)$ & $1565(17.3)$ & \\
\hline \multicolumn{7}{|c|}{ No of attempts at defibrillation: } \\
\hline 0 & $34(1.7)$ & $54(2.7)$ & \multirow[t]{3}{*}{$2.0(0.35)$} & $8368(92.4)$ & $8214(90.7)$ & \multirow[t]{3}{*}{$3.4(3.98)$} \\
\hline $1-2$ & $984(49.4)$ & $978(49.1)$ & & $558(6.2)$ & $637(7.0)$ & \\
\hline$\geq 3$ & $972(48.8)$ & $958(48.1)$ & & $132(1.5)$ & $207(2.3)$ & \\
\hline \multicolumn{7}{|c|}{ EMS call-contact interval (min): } \\
\hline$\leq 6$ & $578(29.0)$ & $561(28.2)$ & \multirow[t]{4}{*}{$2.5(0.48)$} & $2069(22.8)$ & 2075 (22.9) & \multirow[t]{4}{*}{$0.4(0.13)$} \\
\hline $7-8$ & $642(32.3)$ & $634(31.9)$ & & $2547(28.1)$ & $2539(28.0)$ & \\
\hline $9-10$ & $401(20.2)$ & $419(21.1)$ & & $2034(22.5)$ & $2046(22.6)$ & \\
\hline$\geq 11$ & $369(18.5)$ & $376(18.9)$ & & $2408(26.6)$ & $2398(26.5)$ & \\
\hline $\begin{array}{l}\text { Arrest had cardiac } \\
\text { origin }\end{array}$ & $1828(91.9)$ & $1854(93.2)$ & - & $5820(64.3)$ & $6151(67.9)$ & - \\
\hline $\begin{array}{l}\text { Family member was } \\
\text { bystander }\end{array}$ & $1312(65.9)$ & $1233(62.0)$ & - & $6507 \dagger(71.8)$ & $6324(69.8)$ & - \\
\hline \multicolumn{7}{|c|}{ CPR (EMS)-defibrillation interval (min): } \\
\hline No defibrillation & $34(1.7)$ & $54(2.7)$ & - & $8368(92.4)$ & $8214(90.7)$ & - \\
\hline $1-2$ & $1191(59.8)$ & $1193(59.9)$ & - & $44(0.5)$ & $35(0.4)$ & - \\
\hline $3-4$ & 535 (26.9) & $542(27.2)$ & - & $65(0.7)$ & $65(0.7)$ & - \\
\hline$\geq 5$ & 230 (11.6) & $201(10.1)$ & - & $581(6.4)$ & $743(8.2)$ & - \\
\hline
\end{tabular}


Table 2 (continued)

\begin{tabular}{|c|c|c|c|c|c|c|}
\hline & \multicolumn{3}{|c|}{ VF/VT $(n=3980)$} & \multicolumn{3}{|c|}{ Non-VF/VT ( $n=18$ 116) } \\
\hline & $\begin{array}{l}\text { No adrenaline } \\
(n=1990)\end{array}$ & Adrenaline ( $n=1990)$ & $\begin{array}{l}\text { Patient } \\
\text { imbalance } \\
\% \text { IP (D) }\end{array}$ & $\begin{array}{l}\text { No adrenaline } \\
(n=9058)\end{array}$ & Adrenaline $(n=9058)$ & $\begin{array}{c}\text { Patient } \\
\text { imbalance \%IP } \\
\text { (D) }\end{array}$ \\
\hline$\leq 17$ & $260(13.1)$ & $190(9.5)$ & - & $1092(12.1)$ & $785(8.7)$ & - \\
\hline $18-22$ & $463(23.3)$ & $419(21.1)$ & - & $1965(21.7)$ & $1850(20.4)$ & - \\
\hline $23-29$ & $651(32.7)$ & $673(33.8)$ & - & $2902(32.0)$ & $3110(34.3)$ & - \\
\hline$\geq 30$ & $616(31.0)$ & $708(35.6)$ & - & 3099 (34.2) & $3313(36.6)$ & - \\
\hline \multicolumn{7}{|l|}{ Outcomes: } \\
\hline Overall survival & $267(13.4)$ & $338(17.0)$ & - & $214(2.4)$ & $363 \ddagger(4.0)$ & - \\
\hline $\begin{array}{l}\text { Neurologically intact } \\
\text { survival (CPC 1-2) }\end{array}$ & $131(6.6)$ & $132(6.6)$ & - & $38(0.4)$ & $59(0.7)$ & - \\
\hline
\end{tabular}

VFNT=ventricular fibrillation or ventricular tachycardia; $E M S=e m e r g e n c y$ medical service; $C P R=$ cardiopulmonary resuscitation; $A E D=$ automated external defibrillator; $\mathrm{ROSC}=$ return of spontaneous circulation; $\mathrm{CPC}=\mathrm{Glasgow}$-Pittsburgh cerebral performance category score.

*Variables included in propensity score development show imbalance indicators. IP denotes imbalance proportion defined by sum of absolute differences of proportions between groups among all levels in each variable. D denotes standardised difference defined as $\sqrt{ }\left(P_{1}-P_{2}\right)^{\prime} \Sigma^{-1}\left(P_{1}-P_{2}\right)$, where $(k-1)$ dimensional proportion vector $\mathrm{P}_{1}-\mathrm{P}_{2}$ in variable with $\mathrm{k}$ levels is considered to follow $(\mathrm{k}-1)$ dimensional normal distribution $\mathrm{N}_{\mathrm{k}-1}(0, \Sigma)$. Here, $\mathrm{P} 1$ and $\mathrm{P} 2$ are $(\mathrm{k}-1)$ dimensional proportion vectors of variable with $\mathrm{k}$ levels in VF/VT and non-VF/VT groups, respectively. $\Sigma$ is $(k-1)$ dimensional diagonal matrix with (i,i) element being variance of $\mathrm{i}$-th element $(i=1,2, \ldots, k-1)$ of proportion vector $P_{1}-P_{2}$.

†Data missing in one patient.

‡Data missing in one patient. 
Table 3| Logistic regression analyses among matched patients. Odds ratios for comparison between patients with out of hospital cardiac arrest who received pre-hospital adrenaline (epinephrine) administered by emergency medical services and controls

\begin{tabular}{|c|c|c|}
\hline & \multicolumn{2}{|c|}{ Odds ratio $(95 \% \mathrm{Cl})$} \\
\hline & Unadjusted $^{*}$ & Adjusted $†$ \\
\hline \multicolumn{3}{|c|}{ Ventricular fibrillation/ventricular tachycardia } \\
\hline Overall survival & $1.34(1.12$ to 1.60$) \ddagger$ & 1.36 (1.13 to 1.63$)$ \\
\hline Neurologically intact survival & $1.01(0.78$ to 1.30$) \S$ & $1.02(0.78$ to 1.33$)$ \\
\hline \multicolumn{3}{|c|}{ Non-ventricular fibrillation/ventricular tachycardia } \\
\hline Overall survival & $1.72(1.45$ to 2.04$)$ ฯ & 1.78 (1.49 to 2.13$)$ \\
\hline Neurologically intact survival & $1.57(1.04 \text { to } 2.37)^{\star \star}$ & 1.55 (0.99 to 2.41$)$ \\
\hline
\end{tabular}

*Bivariate analysis after propensity score matching.

†Adjusted for presumed cause (cardiac/non-cardiac origin), time from onset of CPR by emergency medical services to hospital arrival, and type of bystander (family/non-family), and time from onset of CPR to first defibrillation with multivariate logistic regression model.

$\ddagger$ Power $=0.87$.

§Power not calculated because OR was nearly 1.

IPower $>0.99$

**Power $=0.50$. 


\section{Figure}

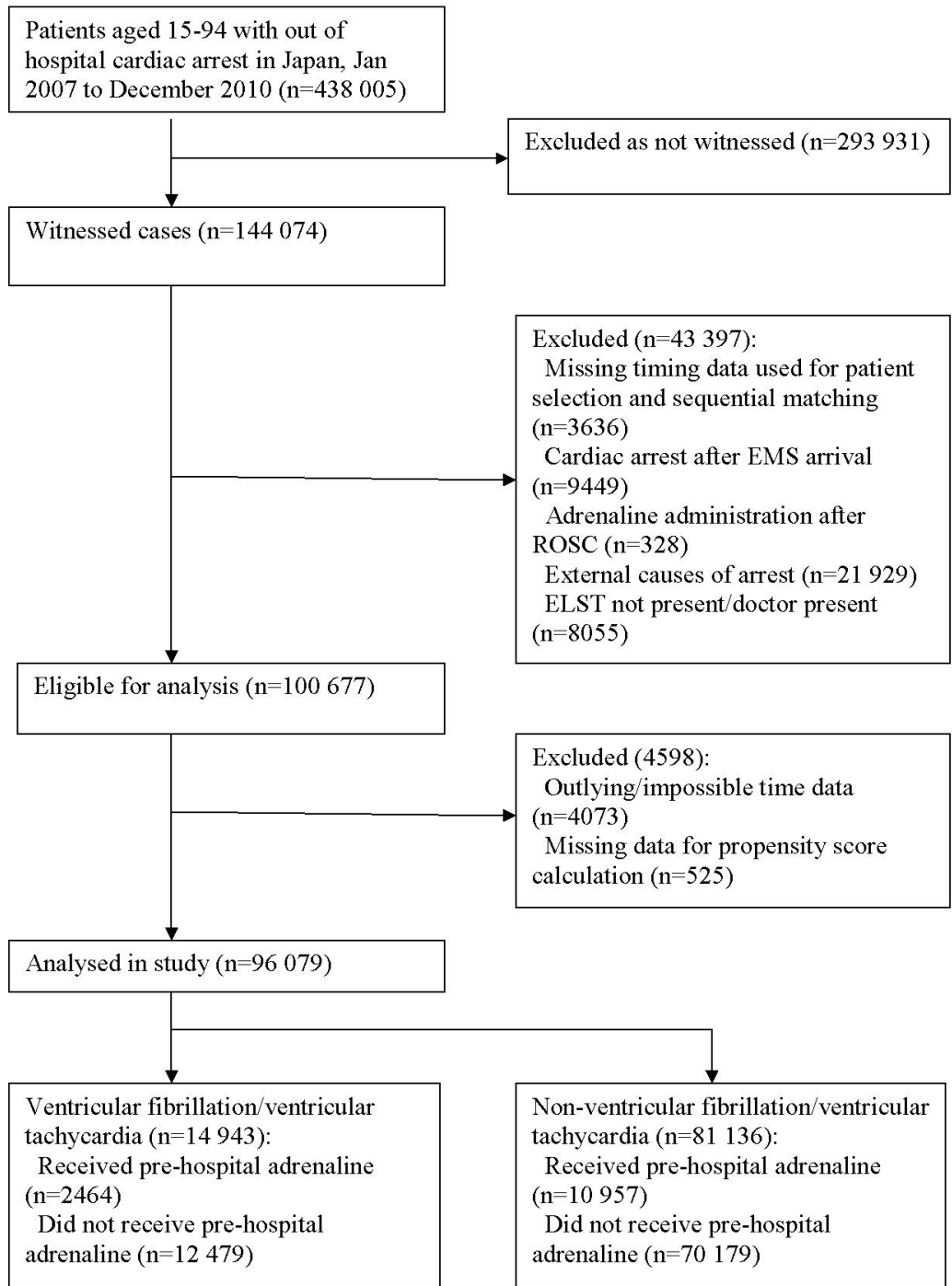

Selection of cases for study of effect of pre-hospital administration of adrenaline (epinephrine) in patients with out of hospital cardiac arrest. EMS=emergency medical service; ROSC=return of spontaneous circulation; ELST=emergency lifesaving technician 